\title{
Concha bullosa, nasal septal deviation, and their impacts on maxillary sinus volume among Emirati people: A cone-beam computed tomography study
}

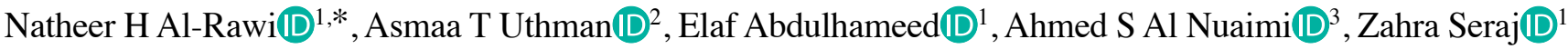 \\ ${ }^{1}$ Department of Oral and Craniofacial Health Sciences, College of Dental Medicine, University of Sharjah, Sharjah, United Arab Emirates \\ ${ }^{2}$ Department of Dental Surgical Sciences, College of Dentistry, Gulf Medical University, Ajman, United Arab Emirates \\ ${ }^{3}$ Department of Clinical Research-Clinical Affairs Directorate, Primary Health Care Corporation (PHCC), Doha, Qatar
}

\section{ABSTRACT}

Purpose: To determine the prevalence of concha bullosa (CB) and nasal septal deviation (NSD) and their impact on maxillary sinus volume (MSV).

Materials and Methods: Cone-beam computed tomographic (CBCT) images of 106 Emirati people were used in this study. The direction and angle of septal deviation were calculated. The presence of $\mathrm{CB}$, which could be unilateral, contralateral, or bilateral in relation to the direction of NSD, was also recorded. MSV was measured using reconstructed Digital Imaging and Communication in Medicine images on Dolphin 3D imaging software version 11.8 premium (Dolphin Imaging, Chatsworth, CA, USA). $P$ values $<0.05$ were considered to indicate statistical significance.

Results: CB was detected in $37.7 \%$ of the sample; $20.7 \%$ of the sample showed single unilateral CB and $16.6 \%$ had single bilateral CB. NSD was seen in $74.5 \%$ of the sample. In the participants with CB, $45.5 \%$ showed mild deviation, $34.4 \%$ showed moderate deviation, and only $12.5 \%$ showed severe septal deviation. CB, but not NSD, was associated with significantly higher MSV on the affected side $(P=0.001)$.

Conclusion: Although NSD was observed in more than two-thirds of the sample and CB was present in more than one-third of the sample, only CB had a significant impact on MSV.(Imaging Sci Dent 2019; 49: 45-51)

KEY WORDS: Turbinate; Nasal Septum; Maxillary Sinus; Cone-Beam Computed Tomography

\section{Introduction}

The anatomic abnormalities and pathological conditions of the sino-nasal complex can be easily identified using computed tomography (CT) ${ }^{1,2}$ As cone-beam CT (CBCT) has become more widespread, features of the nose and paranasal sinuses have become more easily identified by oral radiologists, with higher accuracy. ${ }^{3}$ In the osteo-meatal complex region, concha bullosa $(\mathrm{CB})$ is considered the most common anatomic variation. ${ }^{4}$ It can be simply described as a pneumatized middle turbinate. The causes of pneumatization are unclear, although trauma, nasal septal

Received October 14, 2018; Revised December 13, 2018; Accepted December 18, 2018 *Correspondence to: Prof. Natheer H Al-Rawi

Department of Oral and Craniofacial Health Sciences, College of Dental Medicine, University of Sharjah, PO Box: 27272, Sharjah, United Arab Emirates

Tel) 971551100169, E-mail) nhabdulla@sharjah.ac.ae deviation (NSD), and mouth breathing have been established as predisposing factors of $\mathrm{CB} .{ }^{5}$ Earwaker reported a prevalence of $\mathrm{CB}$ of $55 \%$ of his sample, ${ }^{6}$ whereas Zinreich et al. ${ }^{7}$ reported that $\mathrm{CB}$ was present in $34 \%$ of their sample. $\mathrm{CB}$ can compromise the middle meatus, particularly the osteo-meatal complex, which in turn may make maxillary sinus disease more likely due to the compromised mucociliary drainage. ${ }^{3,7}$ Although the majority of cases of $\mathrm{CB}$ are asymptomatic, ongoing research is being conducted into its association with sinus disease, when the osteo-meatal complex is blocked. ${ }^{8,9}$

NSD is a very common problem among individuals from the Middle East. ${ }^{10}$ The most common reported cause of septal deformity is trauma during infancy and childhood. ${ }^{5}$ However, there is little evidence regarding the possibility that NSD or pneumatization of the middle turbinate may act as a potential contributor to MSV changes and sub-

Copyright (c) 2019 by Korean Academy of Oral and Maxillofacial Radiology

This is an Open Access article distributed under the terms of the Creative Commons Attribution Non-Commercial License (http://creativecommons.org/licenses/by-nc/3.0) which permits unrestricted non-commercial use, distribution, and reproduction in any medium, provided the original work is properly cited. Imaging Science in Dentistry · pISSN 2233-7822 eISSN 2233-7830 
sequent sinus pathology. In the literature, NSD and CB have been found to interfere with proper air flow, which in turn may increase an individual's predisposition to sinus disease, but the relationship of this phenomenon with volumetric changes of the maxillary sinuses remains to be clarified. ${ }^{11}$ The objective of this study was to determine the prevalence of CB, NSD, and sinus pathology in a selected sample of Emirati individuals and to explore the possible association of these findings with MSV changes.

\section{Materials and Methods}

The data collection for this retrospective study was approved by the Medical Ethics Committee of the University of Sharjah, United Arab Emirates (UAE). A total of 106 maxillofacial CBCT scans were taken over the course of 2 years (from October 2015 to October 2017) at the College of Dental Medicine, University of Sharjah (Sharjah, UAE). The study sample was limited to Emirati adults over the age of 18 years. Patients' medical records were reviewed to exclude individuals with a positive history of any prior sino-nasal surgery and/or craniofacial trauma.

All scans were taken using the Galileo 3D X-Ray system (Sirona Dental Systems, Long Island City, NY, USA) with a voxel size of $150 \mu \mathrm{m}$. The images were reproduced and observed on the axial, coronal, and sagittal planes, and all scans were reviewed for any abnormalities in the sino-nasal complex, especially CB, NSD, and maxillary sinusitis. The direction of NSD and the angle of deviation were measured on coronal CBCT images. The angle of deviation was defined as "the angle between the crista galli and the most prominent point of deviation". ${ }^{12}$ The convex part of the nasal septum was identified as the direction of deviation. Patients were subsequently split into 3 groups according to the severity of NSD, defined by the angle of deviation: mild (less than $9^{\circ}$ ), moderate $\left(9^{\circ}-15^{\circ}\right.$ ), and severe (greater than $\left.15^{\circ}\right)^{12}$ (Fig. 1). CB were classified according to the direction of NSD as unilateral, contralateral, or bilateral.

In accordance with Rak et al., ${ }^{13}$ maxillary sinusitis was defined as a radiographic mucosal thickening greater than $3 \mathrm{~mm}$. The likelihood of asymmetries arising as a result of incorrect patient positioning and lack of position standardization was considered, and reference lines were used to correct the measurement plane in all patients. Multiplanar reconstructed coronal and sagittal images were generated and reviewed, and the greatest dimensions of the maxillary sinus were recorded. Sinus height was measured on coronal reconstructed images, from the lowest point of the sinus floor to the highest point of the sinus roof. Maxillary

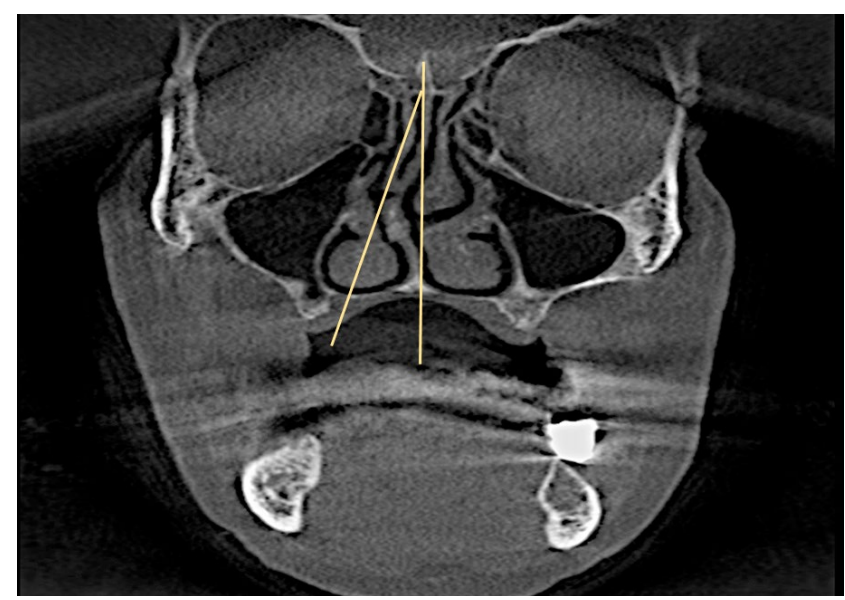

Fig. 1. Measurement of the angle of septal deviation (the angle between the crista galli and the most prominent point of deviation).

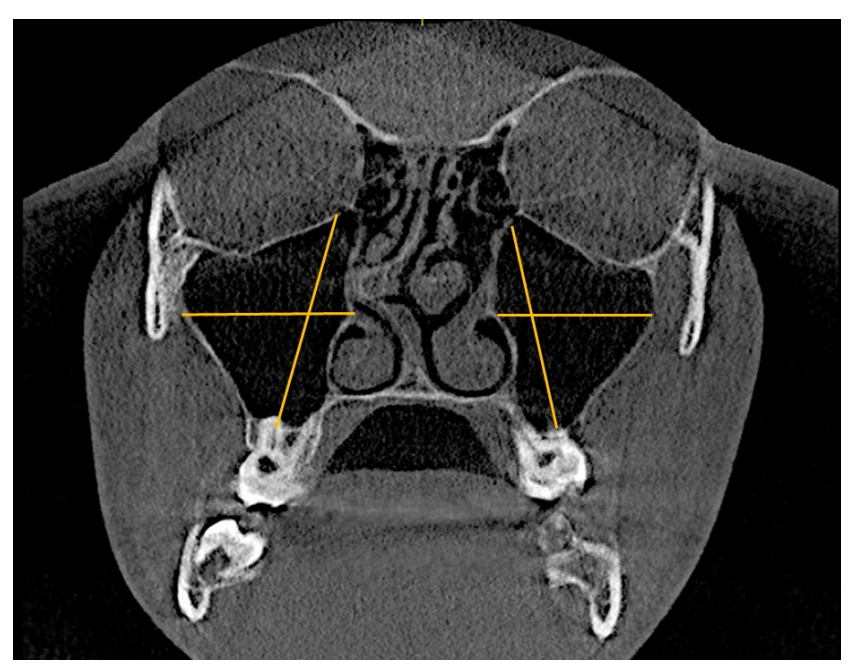

Fig. 2. Measurement of maxillary sinus height (from the lowest point of the sinus floor to the highest point of the sinus roof) and width (from the longest perpendicular distance from the medial wall of the sinus to the outermost point of the lateral wall of the maxillary sinus).

sinus width was measured on coronal reconstructed images as the longest perpendicular distance from the medial wall of the sinus to the outermost point of the lateral wall of the maxillary sinus (Fig. 2). The anteroposterior dimension of the sinus was measured as the longest distance antero-posteriorly from the most anterior point to the most posterior point on sagittal reconstructed images (Fig. 3). MSV was measured using the airway tool of Dolphin 3D imaging software version 11.8 premium (Dolphin Imaging, Chatsworth, CA, USA) from reconstructed Digital Imaging and Communication in Medicine images (Fig. 4). To determine the reproducibility and reliability of the measured variables, intra-examiner calibration was performed by comparing 20 
randomly selected CBCT measurements made by the same radiologist (AU) at a 2-week interval using the paired-sample t-test. No statistically significant difference was noted between the first and second measurements $(P>0.05)$.

SPSS for Windows (version 23.0; IBM Corp., Armonk, NY, USA) was used to perform the statistical analysis.

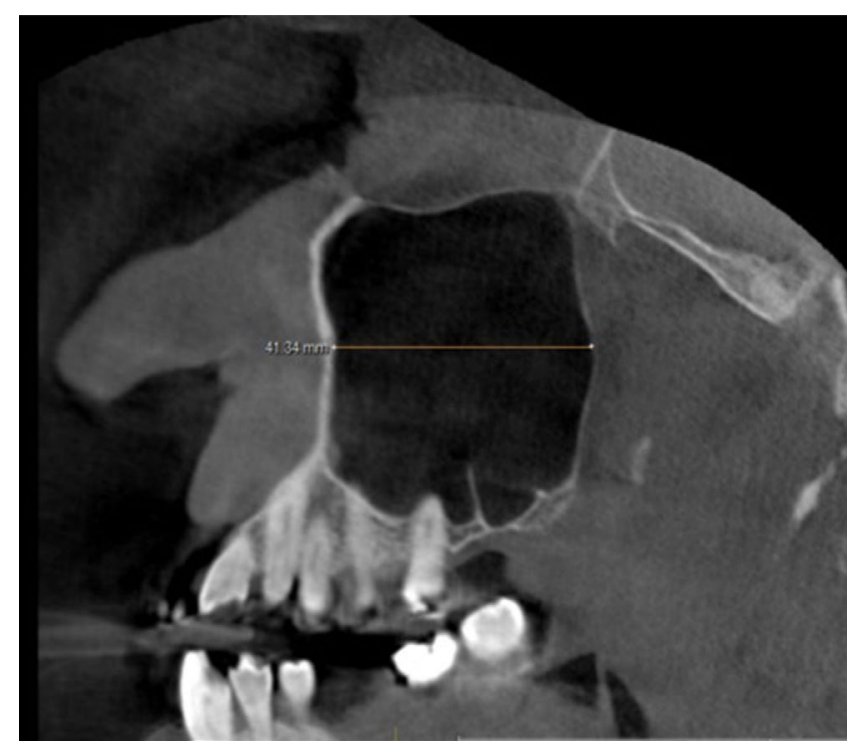

Fig. 3. Measurement of the anteroposterior dimension of the maxillary sinus from the most anterior point to the most posterior point.

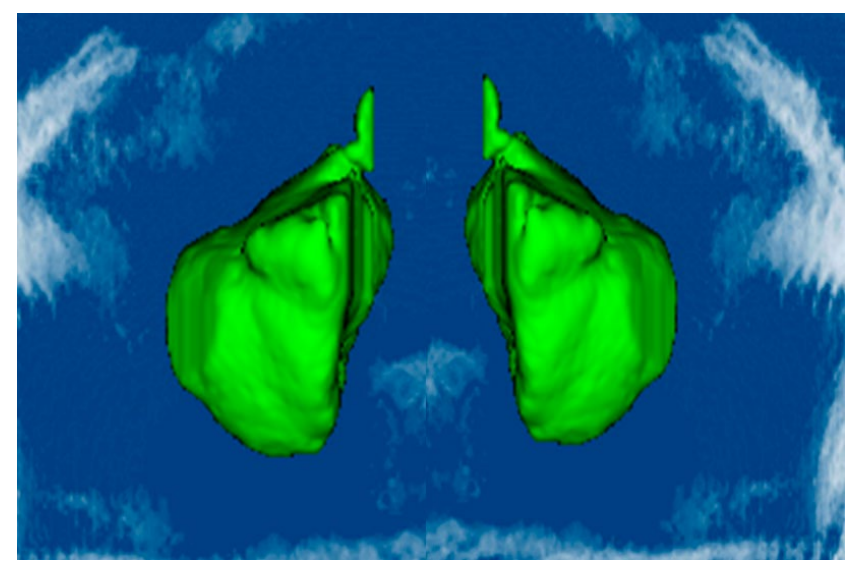

Fig. 4. Reconstructed 3-dimensional image of the maxillary sinuses for volume measurements.
Categorical variables were presented as percentage and frequency, and continuous variables were indicated by mean, standard deviation, range, and their minimum/maximum values. The independent and paired t-tests were also used to compare mean values. A multiple linear regression model was constructed using a backward selection logarithm. For the statistical comparisons that were made, statistical significance was considered to be present at $P<0.05$.

\section{Results}

The average age of the patient population examined was $39.25 \pm 15.61$ years, and the age range was 18-71 years. The mean right and left MSV was $16.30 \pm 12.79 \mathrm{~cm}^{3}$ and $15.05 \pm 7.57 \mathrm{~cm}^{3}$, respectively. The difference between sides was not statistically significant $(P>0.05)$ (Table 1$)$, although males tended to have significantly greater sinus dimensions than females. Nonetheless, the MSV did not show a significant difference according to sex (Table 1). CB was found in $37 \%$ of females and in $38.8 \%$ of males, with no statistically significant sex difference (Fig. 5).

NSD was found in $68.5 \%(n=77)$ of females and $80.8 \%$ of males, with no statistically significant difference by sex. The prevalence of sinus pathology was significantly higher

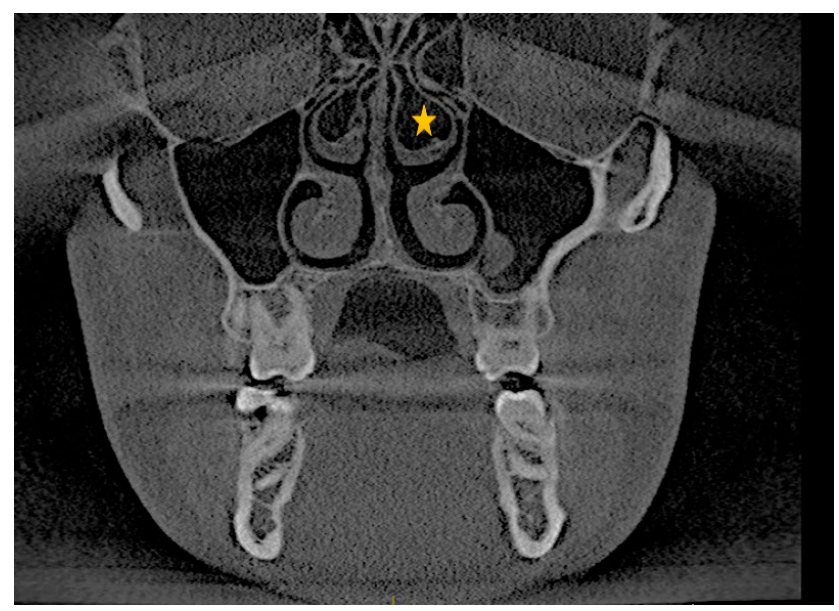

Fig. 5. Bilateral concha bullosa from a coronal slice of cone-beam computed tomography.

Table 1. Differences in maxillary sinus volume according to sex and side

\begin{tabular}{ccccc}
\hline Maxillary sinus arameters & Right & Left & Male & Female \\
\hline Height & $37.5 \pm 5.4$ & $37.8 \pm 5.4$ & $39.83 \pm 5.24$ & $36.67 \pm 4.72^{*}$ \\
Width & $26.7 \pm 4.3$ & $26.7 \pm 4.5$ & $27.61 \pm 4.4$ & $25.88 \pm 4.28^{*}$ \\
Length & $36 \pm 3.4$ & $36 \pm 3.5$ & $36.85 \pm 3.82$ & $35.11 \pm 2.78^{*}$ \\
Volume & $16.30 \pm 12.79$ & $15.05 \pm 7.57$ & $16.52 \pm 10.55$ & $14.71 \pm 10.28$ \\
\hline
\end{tabular}

$* P<0.05$ 
Table 2. Sex differences in the prevalence of concha bullosa, nasal septal deviation, and sinus pathology (number and percentage)

\begin{tabular}{lccc}
\hline & Male & Female & $P$ value \\
\hline Concha bullosa & $20(38.5)$ & $20(37.0)$ & 0.88 \\
Nasal septal deviation & $42(80.8)$ & $37(68.5)$ & 0.148 \\
Sinus pathology & $46(88.5)$ & $35(64.8)$ & $<0.05$ \\
Concha bullosa & & & \\
Absent & $32(61.5)$ & $34(63.0)$ & \\
Unilateral & $8(15.4)$ & $14(25.9)$ & 0.16 \\
Bilateral & $12(23.1)$ & $6(11.1)$ & \\
Sinus pathology & & & \\
Absent & $6(11.5)$ & $19(32.5)$ & \\
Unilateral & $12(23.1)$ & $15(27.8)$ & $<0.05$ \\
Bilateral & $34(65.4)$ & $20(37.0)$ & \\
\hline
\end{tabular}

Table 3. The relative frequency of concha bullosa, nasal septal deviation, and sinus pathology (number and percentage)

\begin{tabular}{clrr}
\hline & & Present & Absent \\
\hline Concha bullosa & & & \\
Nasal septal deviation & Present & $32(40.5)$ & $47(59.5)$ \\
& Absent & $8(29.6)$ & $19(70.4)$ \\
Septal deviation severity & Mild & $15(45.5)$ & $18(54.5)$ \\
& Moderate & $11(34.4)$ & $21(65.6)$ \\
& Severe & $1(12.5)$ & $7(87.5)$ \\
Sinus pathology & Present & $32(80.0)$ & $49(74.2)$ \\
& Absent & $8(20.0)$ & $17(25.8)$ \\
Sinus pathology & & & \\
Nasal septal deviation & Present & $63(79.7)$ & $16(20.3)$ \\
& Absent & $18(66.7)$ & $9(33.3)$ \\
Septal deviation severity & Mild & $28(84.8)$ & $5(15.2)$ \\
& Moderate & $26(81.3)$ & $6(18.8)$ \\
& Severe & $5(62.5)$ & $3(37.5)$ \\
\hline & & &
\end{tabular}

in females $(64.8 \%)$ than in males $(88.5 \%)$ (Table 2). Overall, the prevalence of bilateral $\mathrm{CB}$ was higher in males than in females ( $23.1 \%$ and $11.1 \%$, respectively), while a higher frequency of unilateral $\mathrm{CB}$ was detected in females than in males (25.9\% and $15.4 \%$, respectively); however, this difference was not significant. Bilateral sinus pathology was more common in males $(65.4 \%)$ than in females $(37 \%)$, while an almost equal proportion $(\sim 25 \%)$ of unilateral sinus pathology was observed in both sexes.

Although the relative frequency of CB was clearly higher among individuals with NSD (40.5\%) than among those without NSD (29.6\%), the association between CB and NSD was not statistically significant $(P>0.05)$ (Table 3$)$. Similarly, there was no significant relationship between NSD severity and the prevalence of $\mathrm{CB}$, although it was noted that individuals with mild NSD had a higher rate of CB $(45.5 \%)$ than those with severe NSD (12.5\%).
Table 4. Maxillary sinus volume in relation to the study parameters (unit: $\mathrm{mm}^{3}$ )

\begin{tabular}{lll}
\hline & & Maxillary sinus volume \\
\hline Age (years) & $<30$ & $14.9 \pm 3.9$ \\
& $30-49$ & $16.5 \pm 11.9$ \\
Sex & $>50$ & $15.4 \pm 13.6$ \\
& Male & $16.5 \pm 10.6$ \\
Nasal septal deviation & Female & $14.7 \pm 10.3$ \\
& Present & $15.7 \pm 11.7$ \\
Nasal septal deviation & Absent & $15.3 \pm 5.2$ \\
severity & Mild & $14.7 \pm 12.6$ \\
& Moderate & $17.0 \pm 12.7$ \\
Concha bullosa* & Severe & $14.9 \pm 4.4$ \\
& Present & $14.4 \pm 16.0$ \\
Sinus pathology & Absent & $18.5 \pm 6.7$ \\
& Present & $16.4 \pm 12.6$ \\
& Absent & $14.2 \pm 4.2$ \\
\hline
\end{tabular}

$* P<0.05$

The relative frequency of detected sinus pathology was higher among individuals with NSD (79.7\%) than among those without NSD (66.7\%), but the association was not statistically significant. Likewise, although this tendency was not significant, those with mild NSD had a higher frequency of sinus pathology $(84.8 \%)$ than those with severe NSD (62.5\%) (Table 3). The presence of middle CB had no statistically significant association with the presence of sinus pathology.

Table 4 shows the impact of several studied parameters on MSV. Only the presence of middle CB was associated with a significantly higher MSV.

The presence or absence of NSD did not affect MSV, although the sinus size appeared smaller on the deviated side than on the non-deviated side. Bilateral CB was linked to a larger MSV in comparison to cases without $\mathrm{CB}$ or with only unilateral $\mathrm{CB}$, and the MSV was significantly higher in the presence of $\mathrm{CB}$ (Table 5).

\section{Discussion}

CBCT is a cost-effective method that is being used with increasing frequency for the diagnosis of maxillofacial structures. ${ }^{14}$ Maxillofacial and oral radiologists should be familiar with the sino-nasal complex region. The sino-nasal cavity is conventionally imaged using CBCT exams of the jaws. However, some anatomic variations may lead to chronic sinusitis or complications during sinus surgery, including procedures such as sinus lift surgery, which is performed prior to dental implant placement. Thus, a meticulous understanding of the anatomy of the sino-nasal 
Table 5. Maxillary sinus volume according to the direction of septal deviation and presence or absence of concha bullosa (unit: $\left.\mathrm{cm}^{3}\right)$

\begin{tabular}{|c|c|c|c|c|}
\hline & & Right maxillary sinus & Left maxillary sinus & $P$ value \\
\hline \multirow[t]{6}{*}{ Septal deviation direction } & Straight & $16.28 \pm 5.44$ & $15.07 \pm 4.32$ & 0.14 \\
\hline & Right deviation & $14.96 \pm 5.02$ & $14.61 \pm 5.25$ & 0.64 \\
\hline & Left deviation & $18.05 \pm 19.9$ & $15.32 \pm 11.07$ & 0.32 \\
\hline & Both & $13.76 \pm 3.39$ & $13.47 \pm 3.53$ & 0.81 \\
\hline & $F$ value & 1.505 & 0.625 & \\
\hline & $P$ value & 0.208 & 0.601 & \\
\hline \multirow[t]{6}{*}{ Concha bullosa } & Absent & $14.04 \pm 4.57$ & $14.42 \pm 4.31$ & 0.18 \\
\hline & Dominant right & $21.2 \pm 5.79$ & $21.05 \pm 2.7$ & 0.80 \\
\hline & Dominant left & $16.59 \pm 5.11$ & $16.40 \pm 3.96$ & 0.91 \\
\hline & Bilateral & $16.76 \pm 4.87$ & $17.34 \pm 5.33$ & 0.19 \\
\hline & $F$ value & 6.68 & 7.15 & \\
\hline & $P$ value & $<0.05$ & $<0.05$ & \\
\hline
\end{tabular}

complex is of great importance for minimizing and/or eliminating surgical complications. ${ }^{15} \mathrm{NSD}$ is the most common anatomical variation of the nasal cavity, and it was reported by Earwaker that "NSD occurs in $44 \%$ of individuals with a slight right-side predominance and equal gender distribution." In the present study, more than two-thirds (79.9\%) of the studied sample had NSD, primarily mild or moderate $\operatorname{NSD}(45.5 \%$ and $34.4 \%$, respectively). Deviation of the septum may exacerbate snoring or obstruct breathing if it is severe enough that the septum contacts the lateral nasal wall. In the present investigation, severe NSD was only seen in $7.5 \%$ of the studied sample. Shin suggested that the development of unilateral sinusitis might be influenced by NSD. ${ }^{16}$ According to a meta-analysis conducted by Collet et al., there was "no relationship between the degree of deviation and the presence of sinusitis." ${ }^{, 17}$ Kapusuz et al. reported that the MSV was influenced by severe septum deviation and suggested that the MSV was usually smaller on the deviated side than on the opposing side. ${ }^{18}$ This tendency was also suggested by Lee et al., and is in agreement with the present findings of this study. ${ }^{19}$ Moorthy et al. demonstrated that an S-shaped NSD was significantly correlated with sinus diseases, including cases where nasal symptoms were absent. ${ }^{20}$ Erkan et al. ${ }^{21}$ suggested that both NSD and CB might affect each other physically. In the literature, few studies were found that sought to assess the correlation between NSD and the presence of middle turbinate pneumatization; however, a study demonstrated a highly significant correlation between the presence of middle CB and a deviated septum on the contralateral side. ${ }^{18}$ It has been suggested that the abnormal space created in the nasal septum by the concave part may provide conditions suitable for pneumatization of the middle turbinate. ${ }^{22}$ NSD was usually accompanied with a dominant large $\mathrm{CB} .^{22,23} \mathrm{In}$ contrast, in this study, only $40.5 \%$ of the individuals with NSD had CB, suggesting there may not be an association. Some investigators have suggested that there may be a relationship between having $\mathrm{CB}$ and sinusitis, but other reports found no such relationship. ${ }^{16,24,25}$ In this study, positivity for both NSD and CB had no significant impact on the presence or absence of sinus pathology $(P>0.05)$. The exact mechanism of $\mathrm{CB}$ formation is not yet clear, but the prevalence of middle $\mathrm{CB}$ has been reported to range from $13 \%$ to $72.2 \%{ }^{3,5,26} \mathrm{CB}$ may originate from unilateral or bilateral "variation in the pneumatization of the bone plate caused by an extension of ethmoid cells." ${ }^{27}$ Miranda et al. ${ }^{27}$ suggested that "the degree of pneumatization varies and may cause obstruction of the middle meatus or infundibulum." In the present study, middle CB was observed in $37.7 \%$ of the sample; $20.7 \%$ of the sample had single unilateral CB, and $16.6 \%$ had single bilateral $\mathrm{CB}$. This differs from the findings described in previous studies, ${ }^{24,28}$ and according to the study conducted by Subramanian et al., ${ }^{29}$ the prevalence of $\mathrm{CB}$ among females was higher than among males. However, in the present study, $\mathrm{CB}$ was detected in both sexes equally, with single unilateral $\mathrm{CB}$ being the most common variant among females. Abnormalities of the concha may predispose individuals to osteo-meatal obstruction, which may lead to chronic sinusitis. ${ }^{29,30}$ There also seems to be a connection between increased MSV and CB. Additionally, the sinus volume in the presence of bilateral and/or dominant CB was slightly larger than when $\mathrm{CB}$ was not present. This could be attributed to the intensified growth of the maxillary sinus as a result of inadequate ventilation secondary to the presence of CB. Smith et al. reviewed 883 CT scans, in order to determine the prevalence of $\mathrm{CB}$ and NSD and their possible association with maxillary sinus morphology, and failed to find a significant relationship 
between them. ${ }^{31}$ Similarly, Göçmen et al. ${ }^{32}$ reviewed 300 CBCT scans, were not able to demonstrate "a definite role for NSD, CB and Haller's cells on sinus pneumatization," and suggested the need for further studies in order to clarify the relationship among these phenomena. In the present study, maxillary sinus asymmetry was not affected by the presence of CB and/or NSD, which is in agreement with the study conducted by Demir et al. ${ }^{33}$ Uygur et al. ${ }^{28}$ suggested that "the NSD angle plays an important role on the pneumatization of the concha on the opposite side." In the present study, a non-statistically significant difference was observed between the angle of deviation and the direction of CB $(P<0.05)$. Kucybata et al. ${ }^{34}$ retrospectively analyzed 214 paranasal sinus $C T$ scans in order to investigate whether positivity for NSD and CB was associated with the occurrence of maxillary sinusitis, and concluded that only NSD was related to maxillary sinusitis. Additionally, they demonstrated that bilateral CB had an effect on MSV, which agrees with the present study.

The prevalence and distribution of $\mathrm{CB}$ in this study are similar to the findings of studies conducted in Poland and Iran, ${ }^{34,35}$ whereas the prevalence of NSD in this study is similar to the findings of studies conducted in Poland and Portugal. 23,34

In conclusion, NSD and CB were found to be common radiographic findings in asymptomatic Emirati patients. Although NSD was observed in more than two-thirds of the sample and $\mathrm{CB}$ was observed in more than one-third of the sample, only CB had a significant impact on MSV.

\section{References}

1. Uthman AT, Al-Rawi NH, AL-Naaimi AS, Al-Timimi JF. Evaluation of maxillary sinus dimensions in gender determination using helical CT scanning. J Forensic Sci 2011; 56: 403-8.

2. Uthman AT, AL-Rawi NH, Al-Naaimi AS, Tawfeeq AS, Suhail EH. Evaluation of frontal sinus and skull measurements using spiral CT scanning: an aid in unknown person identification. Forensic Sci Int 2010; 197: 124.e1-7.

3. Bolger WE, Butzin CA, Parsons DS. Paranasal sinus bony anatomic variations and mucosal abnormalities: CT analysis for endoscopic sinus surgery. Laryngoscope 1991; 101: 56-64.

4. Tonai A, Baba S. Anatomic variations of the bone in sinonasal CT. Acta Otolaryngol Suppl 1996; 525: 9-13.

5. Aktas D, Kalcioglu MT, Kutlu R, Ozturan O, Oncel S. The relationship between the concha bullosa, nasal septal deviation and sinusitis. Rhinology 2003; 41: 103-6.

6. Earwaker J. Anatomic variants in sinonasal CT. Radiographics 1993; 13: 381-415.

7. Zinreich SJ, Mattox DE, Kennedy DW, Chisholm HL, Diffley DM, Rosenbaum AE. Concha bullosa: CT evaluation. J Comput Assist Tomogr 1988; 12: 778-84.
8. Yiğit O, Acioğlu E, Cakir ZA, Sişman AS, Barut AY. Concha bullosa and septal deviation. Eur Arch Otorhinolaryngol 2010; 267: 1397-401

9. Hatipoğlu H, Çetin M, Yüksel E. Concha bullosa types: their relationship with sinusitis, ostiomeatal and frontal recess disease. Diagn Interv Radiol 2005; 11: 145-9.

10. Alharethy S, Aldrees T, Aljrid R, Alanazi A, Algaryan SK, Jang YJ. Common nasal deformities among rhinoplasty patients in a university hospital in Saudi Arabia. Ann Saudi Med 2017; 37: 2017-211.

11. Wang RG, Jiang SC, Gu R. The cartilaginous nasal capsule and embryonic development of human paranasal sinuses. J Otolaryngol 1994; 23: 239-43.

12. Serifoglu I, OZ III, Damar M, Buyukuysal M, Tosun A, Tokgöz O. Relationship between the degree and direction of nasal septum deviation and nasal bone morphology. Head Face Med 2017; 13: 3 .

13. Rak KM, Newell JD 2nd, Yakes WF, Damiano MA, Luethke JM. Paranasal sinuses on MR images of the brain: significance of mucosal thickening. AJR Am J Roentgenol 1991; 156: 3814.

14. Al-Rawi NH, Uthman AT, Sodeify SM. Spatial analysis of mandibular condyles in patients with temporomandibular disorders and normal controls using cone beam computed tomography. Eur J Dent 2017; 11: 99-105.

15. Kantarci M, Karasen RM, Alper F, Onbas O, Okur A, Karaman A. Remarkable anatomic variations in paranasal sinus region and their clinical importance. Eur J Radiol 2004; 50: 296-302.

16. Shin HS. Clinical significance of unilateral sinusitis. J Korean Med Sci 1986; 1: 69-74.

17. Collet S, Bertrand B, Cornu S, Eloy P, Rombaux P. Is septal deviation a risk factor for chronic sinusitis? Review of literature. Acta Otorhinolaryngol Belg 2001; 55: 299-304.

18. Kapusuz Gencer Z, Ozkırış M, Okur A, Karaçavuş S, Saydam L. The effect of nasal septal deviation on maxillary sinus volumes and development of maxillary sinusitis. Eur Arch Otorhinolaryngol 2013; 270: 3069-73.

19. Lee DH, Shin JH, Lee DC. Three-dimensional morphometric analysis of paranasal sinuses and mastoid air cell system using computed tomography in pediatric population. Int J Pediatr Otorhinolaryngol 2012; 76: 1642-6.

20. Moorthy PN, Kolloju S, Madhira S, Jowkar AB. Clinical study on deviated nasal septum and its associated pathology. Int $\mathrm{J}$ Otolaryngol Head Neck Surg 2014; 3: 75-81.

21. Erkan SO, Erkan ZA, Tuhanioğlu B, Haytoğlu S, Güney Z. The relationship between septal deviation and concha bullosa. Kulak Burun Bogaz Ihtis Derg 2017; 27: 74-8.

22. Sazgar AA, Massah J, Sadeghi M, Bagheri A, Rasool E. The incidence of concha bullosa and the correlation with nasal septal deviation. B-ENT 2008; 4: 87-91.

23. Stallman JS, Lobo JN, Som PM. The incidence of concha bullosa and its relationship to nasal septal deviation and paranasal sinus disease. AJNR Am J Neuroradiol 2004; 25: 1613-8.

24. Calhoun KH, Waggenspack GA, Simpson CB, Hokanson JA, Bailey BJ. CT evaluation of the paranasal sinuses in symptomatic and asymptomatic populations. Otolaryngol Head Neck Surg 1991; 104: 480-3.

25. Danese M, Duvoisin B, Agrifoglio A, Cherpillod J, Krayenbuhl 
M. Influence of naso-sinusal anatomic variants on recurrent, persistent or chronic sinusitis. X-ray computed tomographic evaluation in 112 patients. J Radiol 1997; 78: 651-7.

26. Arslan H, Aydinlioglu A, Bozkurt M, Egeli E. Anatomic variations of the paranasal sinuses: CT examination for endoscopic sinus surgery. Auris Nasus Larynx 1999; 26: 39-48.

27. Miranda CM, Maranhão CP, Arraes FM, Padilha IG, Farias LP, Jatobá MS, et al. Anatomic variations of paranasal sinuses at multi-slice computed tomography: what to look for. Radiol Bras 2011; 44: 256-62.

28. Uygur K, Tüz M, Doğru H. The correlation between septal deviation and concha bullosa. Otolaryngol Head Neck Surg 2003; 129: 33-6.

29. Subramanian S, Lekhraj Rampal GR, Wong EF, Mastura S, Razi A. Concha bullosa in chronic sinusitis. Med J Malaysia 2005; 60: 535-9.

30. Lee JS, Ko IJ, Kang HD, Lee HS. Massive concha bullosa with secondary maxillary sinusitis. Clin Exp Otorhinolaryngol 2008; 1: 221-3.
31. Smith KD, Edwards PC, Saini TS, Norton NS. The prevalence of concha bullosa and nasal septal deviation and their relationship to maxillary sinusitis by volumetric tomography. Int J Dent 2010; 2010. pii: 404982.

32. Göçmen G, Borahan MO, Aktop S, Dumlu A, Pekiner FN, Göker K. Effect of septal deviation, concha bullosa and Haller's cell on maxillary sinus's inferior pneumatization; a retrospective study. Open Dent J 2015; 9: 282-6.

33. Demir UL, Akca ME, Ozpar R, Albayrak C, Hakyemez B. Anatomical correlation between existence of concha bullosa and maxillary sinus volume. Surg Radiol Anat 2015; 37: 1093-8.

34. Kucybala I, Janik KA, Ciuk S, Storman D, Urbanik A. Nasal septal deviation and concha bullosa - do they have an impact on maxillary sinus volumes and prevalence of maxillary sinusitis? Pol J Radiol 2017; 82: 126-33.

35. Bahemmat N, Hadian H. The frequency of nasal septal deviation and concha bullosa and their relationship with maxillary sinusitis based on CBCT finding. Int J Med Res Health Sci 2016; 5: 152-6. 